$\begin{array}{ll}\text { Italique } & \text { Italique } \\ \text { Poésie italienne de la Renaissance }\end{array}$

XIV | 2011

Varia

\title{
Un sonetto di Góngora: «Descaminado, enfermo, peregrino»
}

José María Micó

\section{(2) OpenEdition}

Journals

\section{Edizione digitale}

URL: http://journals.openedition.org/italique/333

DOI: 10.4000/italique.333

ISSN: 1663-4438

\section{Editore}

Librairie Droz

\section{Edizione cartacea}

Data di pubblicazione: 1 gennaio 2011

Paginazione: 117-132

ISBN: 978-2-600-01536-3

ISSN: 1423-3983

\section{Notizia bibliografica digitale}

José María Micó, « Un sonetto di Góngora: «Descaminado, enfermo, peregrino» », Italique [Online], XIV | 2011, online dal 25 juin 2014, consultato il 30 avril 2019. URL : http://journals.openedition.org/ italique/333 ; DOI : 10.4000/italique.333

Questo documento è stato generato automaticamente il 30 aprile 2019

(c) Tous droits réservés 


\title{
Un sonetto di Góngora: «Descaminado, enfermo, peregrino»
}

\author{
José María Micó
}

1 Luis de Góngora si ammalò gravemente a Salamanca nell'estate del 1593 e nei mesi successivi scrisse dei bellissimi testi che hanno qualcosa a vedere con quella esperienza: un sonetto di ringraziamento al vescovo della città castigliana Jerónimo Manrique; un romance il cui protag nista, appena guarito da «una grave enfermedad», si presenta come esempio di amante condannato a morte dalla crudeltà della sua donna ( «Con mi ejemplo y estas señas, / caballero, caminad; / que ella me condena a muerte,/y yo me voya enterrar»), e, già nel 1594, un sonetto scherzoso ed ingegnoso in cui, con riferimento al soggiorno salmantino, si spaccia per malato d'amore e si paragona con il Lazzaro biblico e con Lazarillo de Tormes, perché è un resuscitato come quello di Betania, e perché, come il pícaro, è stato servo di un cieco:

$$
\begin{aligned}
& \text { Muerto me lloró el Tormes en su orilla, } \\
& \text { en un parasismal sueño profundo, } \\
& \text { en cuanto don Apolo el rubicundo } \\
& \text { tres veces sus caballos desensilla. } \\
& \text { Fue mi resurrección la maravilla } \\
& \text { que de Lázaro fue la vuelta al mundo, } \\
& \text { de suerte que ya soy otro segundo } \\
& \text { Lazarillo de Tormes en Castilla. } \\
& \text { Entré a servir a un ciego, que me envía; } \\
& \text { sin alma vivo, y en un dulce fuego, } \\
& \text { que ceniza hará la vida mía. } \\
& \text { ¡Oh qué dichoso que sería yo luego, } \\
& \text { si a Lazarillo le imitase un día } \\
& \text { en la venganza que tomó del ciego! }
\end{aligned}
$$

2 Non sapremo mai se al di là della malattia fisiologica ci fu un innamoramento vero e proprio, o se queste poesie ricreano soltanto, come sembra più verosimile, l'antica 
aegritudo amoris senza nessuno scopo di fedeltà autobiografica. Infatti Góngora l'aveva già chiarito dieci anni prima, poco più che ventenne, a proposito di un altro topos dell'amore, il segreto, perché il poeta è un «fingidor» (in un modo, però, non molto pessoano):

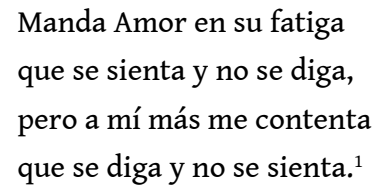

Questo rapporto sempre conflittuale tra vita e poesia è specialmente intrigante nel sonetto che comincia Descaminado, enfermo, peregrino, scritto anch'esso nel 1594: ${ }^{2}$

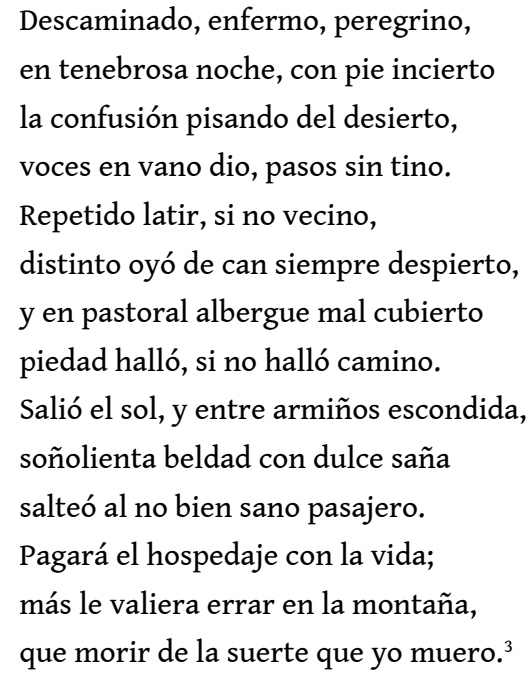

«Dove? Quando? Chi?», si chiedeva giustamente Azorín a proposito della prima quartina. ${ }^{4}$ Il manoscritto Chacón (il codice più importante ed affidabile delle sue poesie) ci offre un titolo molto preciso: «De un caminante enfermo que se enamoró donde fue hospedado», ma altri codici del Seicento, non sempre ben informati e qualche volta un po' troppo fantasiosi, coinvolsero chiaramente l'autore: "A una vez que caminando se perdió»; «Caminando en una noche tempestuosa vino a parar en una casería donde estaba una bellísima dama»; «A una Señora de quien fue hospedado en una casa de campo yendo de camino por allí no bien convalecido de una enfermedad». ${ }^{5}$

Con queste informazioni, non è difficile cadere nella tentazione di risolvere in modo biografico le domande di Azorín, molto pertinenti ed attente alla retorica di base: dove?, a Salamanca; quando?, nell'estate del '93; chi? lo stesso Góngora. Ma c'è un particolare che è tutt'altro che minore: l'autobiografismo amoroso convenzionale, o quanto meno quello esplicito, resta limitato all'ultimo verso, e l'uso della terza per- sona impone un distacco molto originale e rivelatore. Uno dei primi commentatori, García de Salcedo Coronel, propose che il poeta aveva nascosto «en esta ficción el suceso de algún amigo que, desdeñado de su dama, se ausentó y halló en otra parte con nuevo cuidado olvidos para el primero» ('in questa finzione la vicenda di qualche amico che, non corrisposto dalla sua donna, trovò in un'altra parte nuovi affetti e dimenticò quelli di prima'). ${ }^{6}$ In tempi più recenti, è stato suggerito che «los extremos de pasión» del sonetto potrebbero essere soltanto "cortesía para agradecer un generoso hospedaje» ('gentilezza per ringraziare una generosa ospitalità', cosí il grande critico Dámaso Alonso). ${ }^{7}$ A prescin- dere da 
ipotetiche circostanze biografiche, l'esperienza più pertinente qui, come succede spesso nei grandi autori, è quella della tradizione letteraria.

\section{«Perdí mi carrera»: la serranilla}

Quasi cinquanta anni fa, Bruce W. Wardropper mise in rapporto la vicenda del sonetto con «the native Spanish myth of the serranillas", testi per lo più medievali in cui compaiono, fra l'altro, lo smarrimento, i cani abbaiatori, l'albergo, ecc. ${ }^{8}$ Basta il confronto con i primi versi della Serranilla de la Zarzuela.

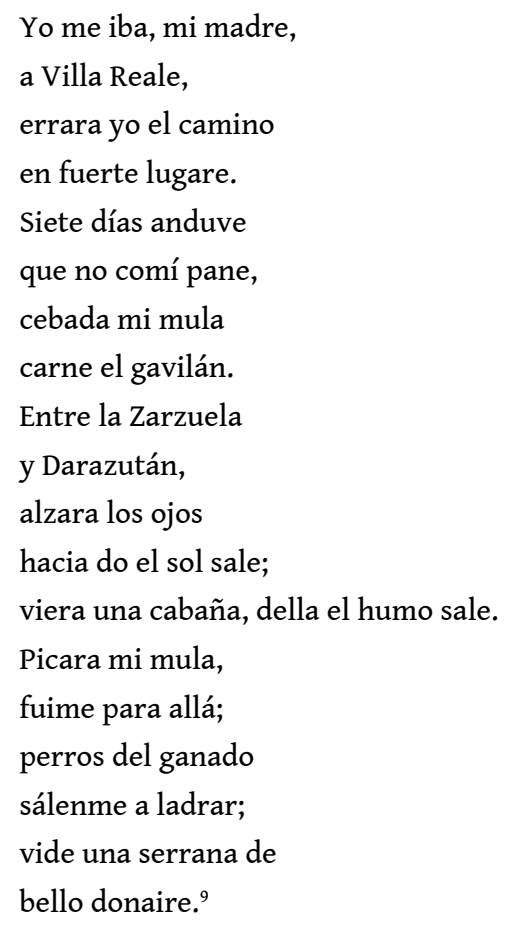

7 Nonostante l'uso del verbo saltear nel sonetto di Góngora (v. 10), che sembra quasi un marchio del genere della serranilla, c'è una differenza essenziale avvertita da Dámaso Alonso: nelle serranillas, «el salteamiento termina en una unión sexual. En el soneto lo que sigue es sólo una nostálgica pena amorosa del salteado». Ma mancano almeno altri tre elementi importanti della pastorella e le sue derivazioni, e la sua assenza mi sembra più significativa di quella dell'unione conclusiva: il dialogo fra i personaggi, l'offerta senza ambiguità della donna, e l'esposizione autobiografica.

Tutt'altro che la vaporosa apparizione della bella di Góngora sono le sfide e le offerte delle serranas: «comamos deste pan duro, / después faremos la lucha», «trota conmigo» ( Libro de buen amor, 969 e 1029); "folgad hora comigo», «entremos a braz partido, / ca dentro en esta espesura / vos quiero luchar dos pares» (Marqués de Santillana); «haremos un hijo» (Serranilla de la Zarzuela). Di certo che il pellegrino del sonetto - «descaminado» 'sviato', non «descamisado» 'scamiciato', come dice un divertente errore di stampa moderno ${ }^{-10}$ non correva un pericolo così tangibile e concreto, e la donna non nascondeva sotto i vestiti delle gambe pelose e delle tette deformi («piernas pelosas» e «tetas disformes») a guisa delle villane del poeta Carvajal. ${ }^{11}$ Nelle serranillas, la cosa più semplice è individuare le circostanze retoriche («Dove? Quando? Chi?»), perché la topografia e l'antroponimia erano caratteristiche del genere: «cerca el Cornejo», «cerca la Tablada» ( 
Libro de buen amor, 993, 1022); «encima de Vozmediano», «camino de Trasovares», «en ese camino / que va a Lozoyuela» (Santillana); «Saliendo de un olivar», «Entre Sesa e Cintura», «Pasando por la Toscana» (Carvajal).

Può darsi che Góngora intendesse preservare, stemperandoli abilmente, alcuni particolari della serranilla, per esempio la paradossale «dulce saña» ('dolce crudeltà', che faceva parte, fra l'altro, delle antitesi tipiche del petrarchismo) con cui lo sfortunato viandante fu assaltato («salteado») dalla sonnolente beltà («soñolienta beldad»), una beltà forse immaginata con i tratti ideali disegnati da uno dei primi poeti italianizzanti del Quattrocento spagnolo, il Marqués de Santillana («más clara que sale en mayo / el alba nin su lucero»), ma che assomiglia soprattutto ad altre figure femminili del grande poeta cordovano: le serranas delle Soledades (I, 233-66: «si Aurora no con rayos, Sol con flores») e quelle di Cuenca del romance «En los pinares de Júcar» (OC, num. 151). Sia quelle che queste, con delle mani bianche («manos blancas») o con pezzi di neve e di madreperla nei piedi («pedazos de nieve y nácar»), destarono la per plessità di un dotto e pignolo amico di Góngora, l'Abad de Rute, «porque no sé que se halle blancura en serranas y gente de playa curtidas al sol y al agua». ${ }^{12}$

Quello che ci conviene ricordare, in ogni caso, è che Góngora, diversamente dalle serranillas ed anche dal suo sonetto del Tormes, decise di togliere tutto quello che era accidentale ed accessorio, assumendo con grande talento ed originalità un altro simbolo centrale della poesia amorosa dell'epoca, precedentemente ricreato in italiano e in spagnolo da due grandi maestri (Petrarca e Garcilaso de la Vega) e da due grandi epigoni (Giovanni Della Casa e Fernando de Herrera).

\section{«Errai gran tempo»: la tradizione petrarchista}

11 Alcuni particolari della prima quartina gongoriana sono il frutto, secondo le informazioni fornite già da Salcedo Coronel, dell'imitazione di certi versi di Giovanni della Casa: il toscano, «misero peregrin», «infermo e stanco peregrino» per un "cammino incerto», dice di essere andato «molti anni [...] con dubbio pie». ${ }^{13}$ Le coincidenze ci mostrano quanto meno che Góngora, soprattutto nei sonetti giovanili degli anni ottanta, rimase vicino al motivo, non proprio modernissimo, del pellegrino amoroso, studiato in Spagna da Antonio Vilanova. ${ }^{14}$ Il motivo risale ovviamente a Petrarca, e partendo dal Canzoniere ( Rvf XXXv, CXXIX, CLXXVI) ebbe grande fortuna in tutta Europa, ma fu Fernando de Herrera «quien ha creado, por influjo de Petrarca, Bembo y Della Casa, la obsesión de la sombra tenebrosa, el caminar con paso incierto, el desierto no tratado, el vagar en confusión perdido, andar errado, e incluso la trimembración característica con que se inicia el soneto de Góngora». ${ }^{15}$ Per scrivere a regola d'arte un poemetto di pellegrinaggio amoroso bisognava che lo smarrimento "por la senda errada» valesse come esempio e come disinganno; così nel sopracitato romance gongoriano «No me bastaba el peligro»o in un sonetto di Quevedo (l'altro grande poeta spagnolo del Seicento), strapieno di reminiscenze petrarchesche:

Si por su mal me sigue ciego amante

(que nunca es sola suerte desdichada),

¡ay!, vuelva en sí y atrás: no dé pisada

donde la dio tan ciego caminante. ${ }^{16}$ 

coetanea ossessione per il motivo della peregrinatio vitae, tutto questo fa ovviamente parte del testo e del contesto del sonetto «Descaminado, enfermo, peregrino», ma non basta a risolvere o cancellare tutte le per plessità che ci provoca nell'insieme. Góngora aveva altro per la testa.

\section{«Voci e passi»}

13 Non è certo una novità fare attenzione al suggerimento di Salcedo Coronel: «Casi parece el mesmo argumento del de las Soledades» ('sembra quasi lo stesso argomento delle Soledades'). ${ }^{17}$ L'intuizione del commentatore è stata accolta dai migliori gongoristi del Novecento, Dámaso Alonso, Antonio Vilanova e Robert Jammes, attenti soprattutto alla somiglianza con «l'inizio della Soledad primera», al «carattere intimo e soggettivo» imposto dal pellegrino d'amore ed alla condivisione dell'ideale bucolico. ${ }^{18} \mathrm{Ci}$ conviene guardare da vicino tutte le coincidenze, verbali e d'argomento, e possiamo chiamare «pellegrino» il protagonista del sonetto e «naufrago» quello delle Soledades. ${ }^{19}$

La prima particolarità condivisa da entrambi i personaggi è che conosciamo le loro vicende tramite un racconto in terza persona. Abbiamo già detto che l'endecasillabo trimembre dell'inizio Descaminado, enfermo, peregrino può essere stato suggerito da Fernando de Her- rera («sin bien, perdido, ajeno de reposo», «sin luz, sin guía, en confusión perdido»), ${ }^{20}$ ma forse Góngora intendeva riunire in un modo meno scontato i tre aspetti dello smarrimento (fisico, amoroso e morale), radunati ancora una volta in un'altro endecasillabo trimembre della Soledad primera: «naúfrago y desdeñado, sobre ausente» (9). Il campo semantico della confusione fa parte dell'ornamento richiesto dai modelli, e non è per niente strano, dunque, il fatto che compaia in modo ossessivo nell'inizio delle Soledades: «En soledad confusa» si sono persi i passi del protagonista e hanno tratto ispirazione i versi del poeta (v. 3 de la dedica); il naufrago intraprende la sua ascensione, illuminata "confusamente» dalla luce crepuscolare, «menos cansado que confuso» (43 e 51). ${ }^{21}$, moltre nella Fabula de Polifemo y Galatea, Góngora ci parla del contadino che rientra al tramonto con i buoi, «pisando la dudosa luz del día» 'calpestando l'incerta luce del giorno' (IX, 8), e il naufrago delle Soledades scala la montagna «entre espinas crepúsculos pisando» (48).

Nella seconda quartina, l'analogia più spiccata tra il sonetto e la silva è l'allusione a un cane i cui abbaî, lontani ma distinti, fungono da guida al pellegrino e al naufrago (84-85):

el can ya, vigilante,

convoca despidiendo al caminante.

Il lettore di oggi può notare l'uso particolare di due parole in accezioni poco comuni (latir $y$ distinto), che diventeranno, però, tipiche dei poemi maggiori di Góngora (Polifemo, xxIV, 2, e Soledades, I, 58), e si ricorderà di quel «can siempre despierto» ('cane sempre sveglio') quando leggerà un altro passaggio della Soledad primera in cui il naufrago, dopo aver mangiato e bevuto le generose ed umili offerte dei pastori, dorme «sobre corchos» un sonno «más regalado» di quelli di un principe «entre holandas», e nella tranquillità notturna, non interrotta dallo scordato strepito delle armi, si sente soltanto un cane ostinato contro con le foglie secche cadute dagli alberi (171-175): 


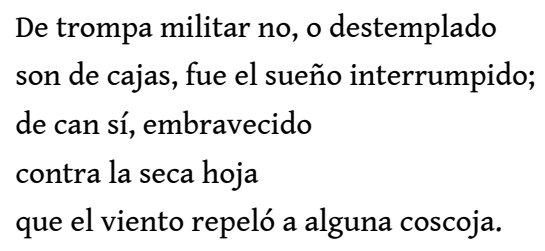

Il «pastoral albergue mal cubierto» è uno degli elementi più caratteristici del paesaggio ideale gongoriano: compare nel Romance de Angélica y Medoro, del 1602 (OC, num. 132) e in una canzone del 1614 (OC, num. 274), e diventa pure un ritornello nella parafrasi del Beatus ille successiva alla salvezza del naufrago (94-135). ${ }^{22}$ C'è un particolare meno rilevante, forse soltanto una curiosità, ma la seconda quartina del sonetto si chiude con un verso bimembre («piedad halló, si no halló camino») che, oltre ad essere un ottimo esempio anticipato di una formula sintattica che finirà per diventare frequente nell'autore (A, si no B) ci ricorda anche il momento in cui il ciclope Polifemo, appacificato secondo lui stesso dall'amore, racconta a Galatea che la sua caverna è «albergue hoy por tu causa al peregrino, / do halló reparo, si perdió camino» (LIV, 7-8). Le coincidenze presentate o insinuate dalla critica finiscono qui, ma c'è dell'altro.

\section{«Una descortesía muy tacaña»}

18 Non sapremo mai a che cosa avesse pensato esattamente Salcedo Coronel quando scrisse che le Soledades ed il sonetto del 1594 avevano «lo stesso argomento», ma comunque tutti abbiamo trascurato il rapporto tra la prima terzina ed un altro episodio - e non dell'inizio - delle Soledades.

\footnotetext{
Salió el sol, y entre armiños escondida, soñolienta beldad con dulce saña salteó al no bien sano pasajero.
}

Quasi tutte le parole e i motivi della terzina compaiono per filo e per segno, sviluppati per bene, in uno dei passi più belli e complessi dell'intera opera di Góngora: l'arrivo degli sposi con le cui «batallas de amor» si chiude la Soledad primera.

Dopo il silenzio della seconda notte - questa volta interrotto soltanto dal travaglio di un taglialegna (687-704) -, il naufrago incontra il «galán novio» e il «venerable padre de la novia». Detto e fatto questo, il poeta se ne disinteressa e ci descrive in più di venti versi l'atteggiamento di «la bella labradora»e il modo in cui reagisce il «forastero» (cioè il naufrago) quando la vede. Ma procediamo in ordine e vediamo l'arrivo di lei (722-726):

$$
\begin{aligned}
& \text { Al galán novio el montañés presenta } \\
& \text { su forastero; luego al venerable } \\
& \text { padre de la que en sí bella se esconde } \\
& \text { con ceño dulce, y, con silencio afable, } \\
& \text { beldad parlera, gracia muda ostenta. }
\end{aligned}
$$

La contadina è una «beldad» che, senza far motto, esprime la sua grazia, garantita dalla retorica della sua bellezza, e il suo atteggiamento è discreto e riservato: «si nasconde» come la donna del sonetto («entre armiños escondida») e fa vedere un «ceño dulce» (una «dulce saña» nel sonetto del 1594). Indipendentemente dalle virtù retoriche dei due 
passaggi (è molto notevole, ad esempio, l'alliterazione della terzina), ${ }^{23}$ l'ossimoro in comune - nonostante si tratti di un particolare appunto comune nella poesia amorosa - ci indica a mio avviso che il Góngora delle Soledades aveva in mente i propri versi di qualche tempo prima. L'assalto del sonetto non ha niente a vedere con un rapporto sessuale, come pensava Wardropper, ${ }^{24}$ ma con l'inaspettata commozione dell'incontro, motivo più elaborato, ancora una volta, nel passo della Soledad primera (732-736):

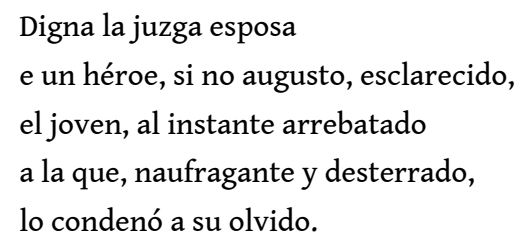

In una parola: appena vede la sposa, così bella, il naufrago pensa nostalgicamente alla sua amata, e poi segue il passo forse «più oscuro e discusso» delle Soledades, ${ }^{25}$ che è come dire uno dei più difficili della letteratura spagnola (737-42):

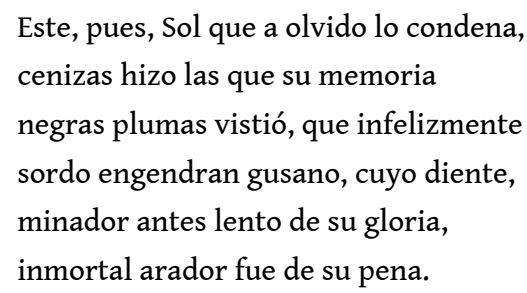

Questo frammento contiene, descritti molto bellamente, «varios afectos propios de un amante» (Díaz de Rivas) e tante difficoltà d'inter pretazione. Il primo ostacolo è l'ambiguità: chi, tra le due donne, è il Sole?; si tratta soltanto di un modo di alludere alla giornata in cui è avvenuto l'incontro?; qual è il tempo di quell'avverbio antes del verso 741?; cosa intende dirci l'autore con l'allusione alla metamorfosi della Fenice?; cosa signifi ca che il naufrago risulta arrebatado a su primer amor?; è polisemico quell'arador (aratore e acaro)? Nonostante tutto, è possibile capire il passaggio in un modo generico: l'estasi per la bellezza della sposa cancella per un attimo le meste ricordanze del naufrago, ma finisce per aggravare senza rimedio la sua malinconia, provocandogli una tristezza così profonda che si sarebbe risolta in pianto («el alma, por los ojos desatada, / señas diera de su arrebatamiento») se non fosse stato per l'opportuno arrivo dei giovani pastori con la loro musica rustica ed allegra (743-54). Il testo delle Soledades esprime, dunque, un sentimento paradossale: «la présence de la fiancée suscite [...] aussi l'absence d'une absente» (Nadine Ly). ${ }^{26}$

Nella seconda obiezione dell'Antídoto contra la pestilente poesía de las "Soledades», Juan de Jáuregui, che non voleva altro che mettere Góngora in ridicolo, disse però una cosa molto interessante a proposito della «traça de esta fábula»: il naufrago «sólo hace una descortesía muy tacaña y un despropósito: que se olvida de su dama ausente, que tantas querellas le costó al salir del mar, y se enamora de esotra labradora desposada en casa de su mismo padre, donde lo hospedaron cortésmente, sin que sirva aquello de nada al cuento, sino para echarlo más a perder» ('il naufrago fa soltanto una sgarbatezza molto meschina e uno sprop sito: dimentica la sua donna assente, quella che gli è costata tante lamentele all'inizio, e s'innamora di quest'altra contadina sposata proprio a casa del suo padre, che è stato per giunta il suo generoso ospite, e tutto questo non serve a niente al racconto, anzi serve a rovinarlo ancora di più') ${ }^{27}$ Riassumendo con tanto di paradosso: il 
titolo del sonetto nel manoscritto Chacón («De un caminante enfermo que se enamoró donde fue hospedado») sarebbe andato benissimo, secondo il parere di Juan de Jáuregui, per spiegare la Soledad primera.

La spiegazione scherzosa dell'Antídoto e tutte le altre difficoltà di questo famoso locus obscurus ci rivelano le due coincidenze principali fra le Soledades e l'endecasillabo salteó al no bien sano pasajero: la malattia d'amore del naufrago (malato in precedenza, per così dire) e la sua commozione di fronte alla bellezza della donna appena conosciuta. Il viandante del 1594 «se enamoró» ('s'innamoró'), e nonostante quello del 1613 non avesse fatto altro che rattristarsi, entrambi hanno una storia affettiva precedente all'incontro con la donna. La malattia che apre il sonetto e la morte (il «morir») che lo chiude non possono avere che un senso amoroso, e per questo Salcedo Coronel intese «no bien sano» come malato «de las memorias de su ingrato dueño», facendo riferimento all'ancora vigente midons trovadorico. ${ }^{28}$

Soltanto alla fine del sonetto compare l'io del poeta, in tono ammonitorio, per condividere con il pellegrino il suo stato d'innamoramento, ma non per forza tutti gli altri particolari dell'avventura. Dopo l'approccio onnisciente e distante del racconto, la sor presa dell'ultimo verso fa aumentare l'ambiguità e imbroglia ancora di più l'inter pretazione della poesia, ma non ci permette di dire che alla fine vanno identificati il pellegrino, il narratore ed il poeta «as one and the same person». ${ }^{29}$

Questo balzo dalla prima alla terza persona e viceversa avrà un trattamento di grande novità in una bellissima canzone scritta nell'anno 1600, e ci mostra che Góngora si rese conto con grande intelligenza del fatto che l'io dei poeti petrarchisti era già guasto e un po' trasandato dopo qualche secolo d'amorose fallacie. Così, alcuni dei suoi testi più avvicinati all'autobiografismo tradizionale riducono alla minima espressione la presenza esplicita della prima persona e affidano la sua espressività, per esempio, alla ricreazione di un motivo mitologico (come nel sonetto «Gallardas plantas...», OC, num. 45) o al paragone fra i «cuidados» dell'amante e le violenze scatenate dalla Natura («Cosas, Celalba mía...», OC, num. 107).

Cosas, Celalba mía, he visto extrañas: cascarse nubes, desbocarse vientos, altas torres besar sus fundamentos y vomitar la tierra sus entrañas; duras puentes romper, cual tiernas cañas, arroyos prodigiosos, rios violentos, mal vadeados de los pensamientos y enfrenados peor de las montañas; los días de Noé, gentes subidas en los más altos pinos levantados, en las robustas hayas más crecidas; pastores, perros, chozas y ganados sobre las aguas vi, sin forma y vidas, y nada temí más que mis cuidados. 


\section{«El momento y la sensación»}

28

Azorín mise in rapporto il sonetto del 1594 con il particolarissimo impressionismo del Greco, valutando «esa sensación prístina e inactual» che nascondono i suoi versi, ma la sua per plessità sarebbe stata maggiore se avesse saputo che i particolari e $\mathrm{i}$ «momenti» di quel «mundo de fantasmagoría, de ensueño y de dolor», tutto irreale e misterioso, ${ }^{30}$ avevano una corrispondenza così precisa, così attenta ai dettagli, con l'«argumento»o la «traça» della Soledad primera.

Non c'è motivo, idea, personaggio, sfumatura del sonetto che non compaia nella silva del 1613: il pellegrino malato d'amore, la confusione del paesaggio, il cane sempre sveglio, l'albergo umile ed ospitale, l'alba, la bella schiva, la commozione, il ricordo dell'amore precedente, la felicità impossibile, la nostalgia... e ovviamente tutta la sfilza di similitudini verbali imposte dalla tradizione. La domanda è molto pertinente: come spiegare il fatto paradossale che le Soledades siano così utili, così necessarie per capire un poemetto scritto quasi vent'anni prima?

Il fatto è che nel sonetto Descaminado, enfermo, peregrino troviamo, con un anticipo di quasi vent'anni, le prime impronte della genesi delle Soledades. Nel contesto della poesia europea, è importante capire che, a dispetto della fedeltà al modello da parte di altri autori, anche molto posteriori (Quevedo, Villamediana, Bocángel), il percorso di Góngora aveva uno scopo molto preciso che si capisce ancora meglio con testi come la canzone Qué de invidiosos montes levantados, che all'inizio non sembra altro che una traduzione del Petrarca, ma rappresenta il definitivo sor passo del petrarchismo, quanto meno all'interno dell'opera de un singolo, ma grandissimo, poeta cordovano del Seicento. Un sonetto perfetto, che è addirittura un abbozzo di quello che l'autore intendeva scrivere un giorno, dopo vent'anni. Forse nel 1594 non era ancora pronto, ma mentre scriveva quel sonetto in apparenza molto petrarchesco, Góngora pensava meno ai modelli del passato che alle sfide del futuro.

\section{NOTE}

1. È il ritornello di una celebre letrilla scritta nel 1583 (Obras Completas = OC, Madrid, Fundación José Antonio de Castro, 2000, vol. 1, pp. 43-45, num. 36).

2. I primi sonetti di LUIS DE GóNGORA (1561-1627) rimandano spesso a Petrarca, Ariosto, Tasso (sia il padre che il figlio) ed altri poeti italiani, ma il caso del sonetto «Descaminado, enfermo, peregrino» è molto diverso da quelli giovanili e più imitativi. Scritto nel 1594, nei suoi versi s'intrecciano diversi fili della tradizione letteraria: le serranillas, la aegritudo Amoris e un solido petrarchismo di base che fu espresso, però, in modo molto originale. Il profilo e le disavventure del «peregrino», la narrazione in terza persona ed altre particolari annunciano, con quasi vent'anni anni di anticipo, alcuni dei tratti principali dell'argomento delle Soledades: lo smarrimento amoroso, l'abbaio del cane sempre sveglio, l'aurora e la bella dama schiva. Questo 
mio contributo è in essenza, con piccoli cambiamenti, la traduzione italiana del terzo capitolo del mio libro De Góngora, Madrid, Biblioteca Nueva, 2001.

3. Si veda il commento di R.P. Calcraft, The Sonnets of Luis de Góngora, Durham, Università di Durham, 1980, pp. 38-42.

4. Azorín, «El momento y la sensación», in Madrid, ed. M. Lacarta, Madrid, Avapiés 1988, p. 175; cfr. anche Id., «Lo fatal», in Castilla, ed. J.L. Gómez, Barcelona, Planeta, 1986, pp. 194-195.

5. Si veda l'edizione critica dei Sonetos a cura di Biruté Ciplijauskaité, Madison, Hispanic Seminary of Medieval Study, 1981, p. 280; ho corretto la prima epigrafe («A una nave...», si dice, con un errore che sarebbe stato idoneo per la mia argomentazione) dopo aver controllato direttamente il codice (Montaner o Pérez de Rivas, Biblioteca de Cataluña, ms. 2056, fol. 145r).

6. Garciá de SAlCedo Coronel Sonetos, cit., p. 300.

7. Dámaso Alonso, Góngora y el «Polifemo», Madrid, Editorial Gredos, 1961, vol. II, p. 150 (e così per tutte le successive citazioni di Dámaso Alonso).

8. Bruce W. Wardropper, «Góngora and the serranilla», Modern Language Notes, LXXVII (1962), pp. 178-81.

9. Ramón Menéndez Pidal, «Serranilla de la Zarzuela» (1905), in Poesía árabe y poesía europea, Madrid, Espasa Calpe, $1973^{6}$, pp. 119-35 (il testo alle pp. 125-26).

10. R. Espinosa Maeso, «Nuevos datos biográficos de Gó ngora», Revista de Filología Española, XLV (1962), pp. 57-87 (l'errore a p. 77).

11. Cfr. CARVAjal, Poesie, ed. Emma Scoles, Roma, Editioni dell'Ateneo 1967, p. 208.

12. FrANCISCO FERNÁNDEZ DE CóRDoBA, Parecer acerca de las «Soledades», ed. Emilio Orozco Díaz, En torno a las "Soledades», Granada, Universidad de Granada 1969, p. 144 (anche qui va corretto qualche errore dell'editore: si veda il codice della Biblioteca de don Bartolomé March, 18/10/11, fol. 144v).

13. Cfr. Salcedo Coronel, Sonetos, cit., p. 301.

14. Antonio Vilanova, «El peregrino de amor en las Soledades de Góngora» (1952) e «Nuevas notas sobre el tema del peregrino de amor» (1974), raccolti tutti e due in Erasmo y Cervantes, Barcelona, Lumen 1989.

15. A. Vilanova, «El peregrino de amor...», cit., p. 454.

16. Quevedo, Obra poética, ed. José Manuel Blecua, Madrid, 1969-1981, num. 478, I, Castalia, p. 661.

17. LUIS DE GóNGORA, Sonetos, cit., p. 300.

18. A. Vilanova, «El peregrino...», cit., pp. 454-55, e R. Jammes, Études sur l'oeuvre poétique de Luis de Góngora y Argote, Bordeaux, Université de Bordeaux 1967, p. 587 (trad. spagnola, Madrid, Castalia, 1987, pp. 492-93).

19. I numeri fanno riferimento ai versi della Soledad primera.

20. Cfr. la Poesía castellana original completa di HERRERA, ed. Cristóbal Cuevas, Madrid, Cátedra 1985, pp. 467 e 781.

21. Lo spiega Nadine Ly nel suo contributo in Homenaje al profesor Antonio Vilanova, Barcelona, Universidad de Barcelona 1989, I, pp. 355-75.

22. A questo proposito sono fondamentali le parole di Robert Jammes, Études, cit., pp. 586-605 (trad. spagnola, pp. 492-507).

23. Nel suo lavoro «La musicalidad del Polifemo», C. C. Smith fece tempo fa un' attenta analisi fonetica di questo sonetto (Revista de Filología Española, XLIV, 1961, pp. 153-54).

24. Si veda la sua antologia della Spanish Poetry of the Golden Age, Nueva York, Appleton 1971, p. 166.

25. Karl Vossler, La soledad en la poesía española, Madrid, Gelo Sáez 1941, p. 141.

26. Nadine Ly, «Poétique et signifiant linguistique. A propos d'un fragment des Soledades», Bulletin Hispanique, LXXXVII (1985), pp. 447-70 (il passo citato è a p. 458); per quanto riguarda le opinioni 
dei commentatori, è molto utile vedere anche le edizioni delle Soledades di Dámaso Alonso (Madrid, Ediciones del Arbol Castalia 1936) e Robert Jammes (Madrid, 1994).

27. JÁUREGUI, Antídoto contra la pestitente poesía de las Soledades, ed. José Manuel Rico García, Sevilla: Universidad de Sevilla, 2002, pp. 87-88.

28. LUIS DE GóngORA, Sonetos, cit., p. 304.

29. R. P. Calcraft, The Sonnets..., cit., pp. 40-41.

30. Si veda Azorín, Castilla, cit., p. 194, e Madrid, cit., p. 175. 